\title{
Pediatría
}

http://www.revistapediatria.org/

DOI: https://10.14295/rp.v54i1.190

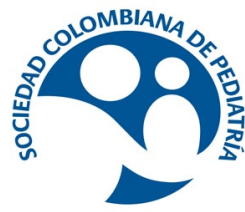

Regional Bogotá

Reportes de caso

\section{Holoprosencefalia de tipo recesivo en una familia endogámica colombiana.}

\section{Luis Gustavo Celis ${ }^{a}$, Isabel Fernández ${ }^{b}$, Humberto Ossac ${ }^{c}$, Andreina Zannin Ferrero ${ }^{a}$, María Camila Garzon ${ }^{a}$, Samantha Mayorga ${ }^{a}$, Luz Ángela Murillo ${ }^{a}$, Paola Gabriela Zuleta ${ }^{a}$.}

a. Facultad de Medicina de la Universidad de la Sabana (Colombia).

b. Centro Médico Docente La Trinidad (Venezuela).

c. Laboratorio de Citogenética y Biología Molecular (Colombia).

INFORMACIÓN DEL ARTÍCULO

\section{Historia del artículo:}

Recibido el 22 abril 2020

Aceptado el 20 agosto 2021

Palabras clave:

Holoprosencefalia.

Neonatología

Labio leporino

Microcefalia

Cariotipo.

Keywords:

Holoprosencephaly

Neonatology

Cleft lip

Microcephaly

Karyotype
R E S U M E N

Antecedentes: La holoprosencefalia es una anomalía congénita cerebral compleja resultante de una división incompleta del prosencéfalo, generando una ausencia total o parcial de la separación de los hemisferios cerebrales, manifestándose clínicamente en diferentes malformaciones craneofaciales. Reporte de caso: En el presente artículo se presenta el caso de una recién nacida femenina, en quien se realizó una historia clínica y examen físico completo, observándose características fenotípicas principales de esta anomalía congénita al igual que muestras sanguíneas y estudios complementarios. Esta patología tiene una etiología heterogénea, variada y son pocos los casos relacionados con un herencia autosómica recesiva por lo que es importante apostar por un diagnóstico temprano prenatal y brindar un asesoramiento multidisciplinario y genético dando una adecuada información a los padres.

Recessive-type holoprosencephaly in a consanguineous Colombian family

A B S T R A C T

Background: Background: Holoprosencephaly is a complex congenital brain anomaly resulting from an incomplete division of the prosencephalon, generating a total or partial absence of separation of the cerebral hemispheres, manifesting itself clinically in different craniofacial malformations. Case report: This article presents the case of a female newborn baby, in whom a complete clinical history and physical examination were performed, observing the main phenotypical characteristics of this congenital anomaly as well as blood samples and complementary studies. This pathology has a heterogeneous and varied etiology, and there are few cases related to an autosomal recessive inheritance. Therefore, it is essential to get on an early prenatal diagnosis and provide multidisciplinary and genetic counseling giving adequate information to the parents.

\footnotetext{
*Autor para correspondencia. Andreina Zannin Ferrero Correo electrónico: andreinazafe@unisabana.edu.co
}

Como Citar: Celis LG, Fernández I, Ossa H, Zannin Ferrero A, Garzón MC, Mayorga S, Murillo LA, Zuleta PG. Holoprosencefalia de tipo recesivo en una familia endogámica colombiana. Pediatr. 2021;54(1):36-39. 


\section{Introducción}

La holoprosencefalia es una anomalía congénita que resulta del fallo en la división del prosencéfalo durante la quinta semana del desarrollo embrionario, generando una ausencia total o parcial de la separación de los hemisferios cerebrales, que se manifiesta con diferentes malformaciones craneofaciales. Tiene una etiología heterogénea y variada y son pocos los casos relacionados con un herencia autosómica recesiva, por lo cual en el presente artículo presentamos el caso clínico de un neonato con holoprosencefalia semilobar de origen autosómico recesivo exponiendo sus hallazgos clínicos, características por imágenes asociadas, factores de riesgo y una breve discusión de la literatura disponible sobre esta anomalía congénita poco frecuente.

\section{Descripción del caso}

Recién nacida de sexo femenino producto de primera gestación de madre de 16 años y padre de 19 años. Padres consanguíneos, primos en segundo grado (Figura No. 1). Gestación sin complicaciones y parto por cesárea. Las medidas antropométricas fueron, peso de 2900 gramos, talla de $35 \mathrm{~cm}$ y perímetro cefálico de $27 \mathrm{~cm}$. Al examen físico, se evidenció microcefalia, hipotelorismo ocular, puente nasal plano, hendidura labial unilateral completa, hendidura palatina completa, pabellones auriculares displásicos de implantación baja rotada, así como también manos con inclusión del pulgar (Figura No. 2). Se toma TAC de cráneo simple donde se evidencian defectos craneofaciales de línea media por falla de la inducción ventral sin apreciación de la cisura interhemisférica, hoz del cerebro en la porción anterior y el cuerpo calloso. En la porción posterior se identificó separación parcial de los lóbulos occipitales y tem- porales. Adicionalmente, se realizó cariotipo en donde se analizaron 30 metafases de alta resolución sin alteraciones estructurales o numéricas, 46,XX. (Figura No. 3).

\section{Discusión}

La Holoprosencefalia (HP) es una anomalía congénita que resulta del fallo en la división del prosencéfalo durante el desarrollo embrionario. Durante la quinta semana de gestación el prosencéfalo se divide horizontalmente en telencéfalo, a partir del cual se originan los hemisferios cerebrales y ventrículos laterales y en diencéfalo, que conlleva la desarrollo de los tálamos, neurohipófisis y tercer ventrículo. El fallo en este proceso embriológico origina la holoprosencefalia, generando una ausencia total o parcial de la separación de los hemisferios cerebrales, manifestándose en diferentes malformaciones craneofaciales, siendo las malformaciones oculares las de mayor incidencia, seguidas por las malformaciones nasales, del oído, boca y paladar (1) tal como se aprecia en el presente caso, que cursa con hipotelorismo ocular, puente nasal plano y hendidura labial unilateral completa y hendidura palatina.

Las malformaciones congénitas del sistema nervioso central ocupan el segundo lugar dentro de las malformaciones congénitas, siendo la holoprosencefalia la malformación craneoencefálica más común. Teniendo un incidencia de 1 por cada $5000 \mathrm{a}$ 10000 nacidos vivos. Sin embargo, tiene una alta mortalidad intrauterina y una incidencia perinatal mucho más elevada, presentándose en 1 en cada 250 embriones $(3,4)$. En Colombia la prevalencia es menor a 1 por cada 100000 nacidos vivos, pero son datos limitada representación epidemiológica, considerando que aún existen casos sin diagnosticar o que no son reportados (5).

Figura 1. Árbol genealógico.

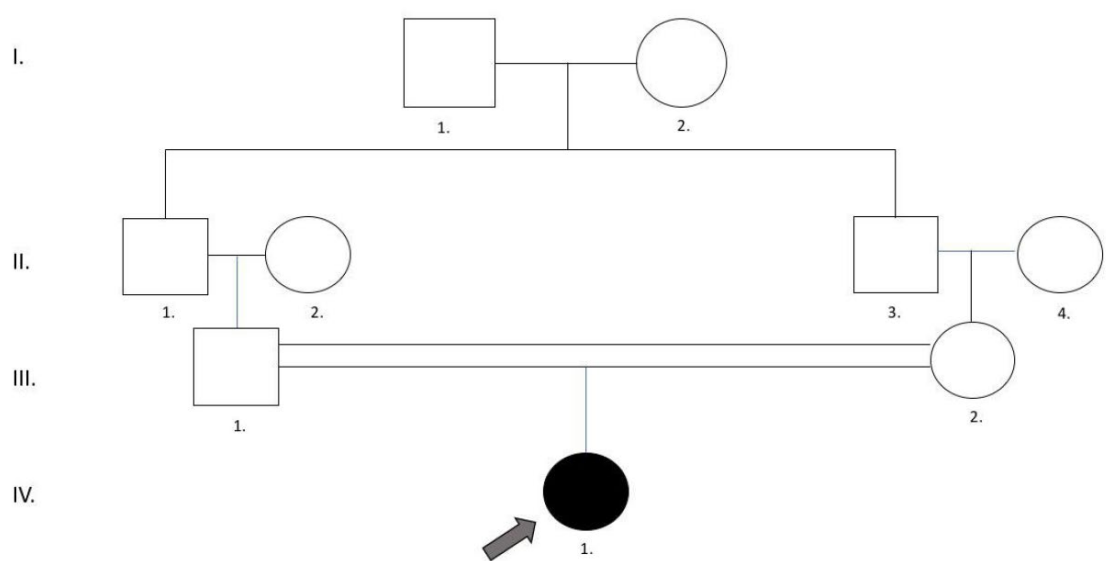

Genealogía de la paciente quien está señalada con una flecha (IIV 1). Se pueden observar la consanguinidad de sus padres. 
Figura 2. Presentación clínica

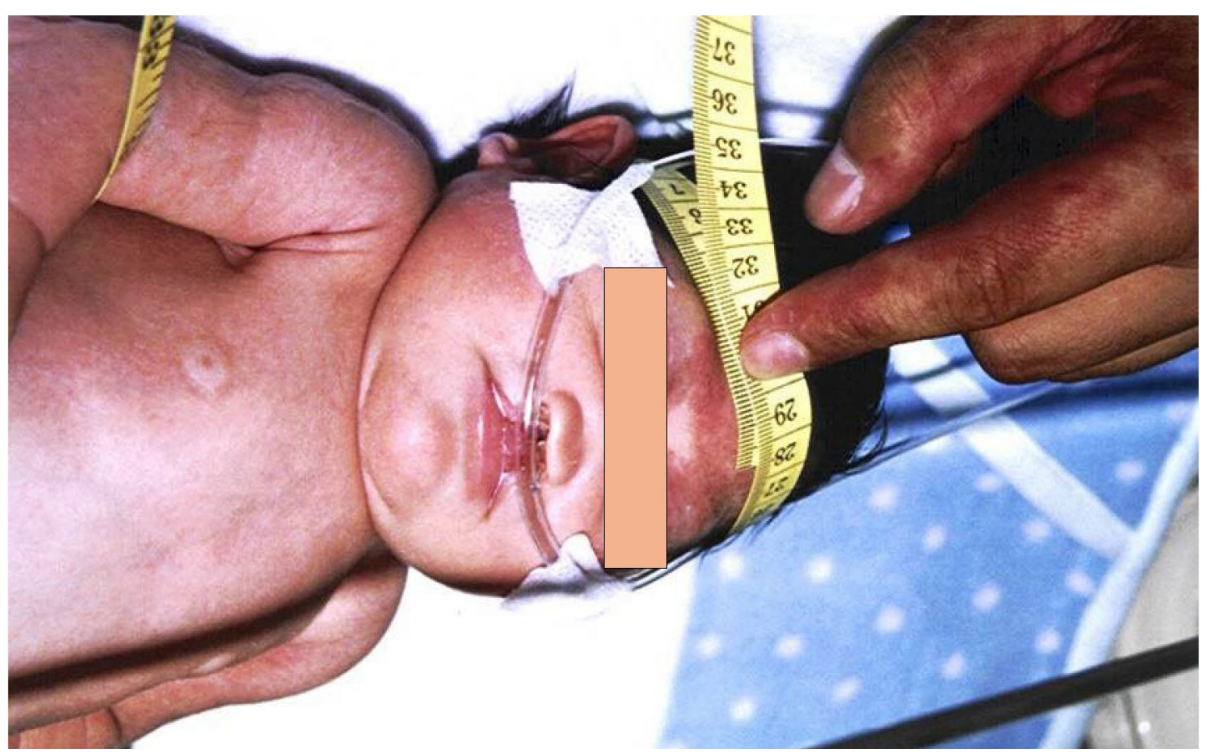

Se observa permito cefálico de $27 \mathrm{~cm}$ compatible con microcefalia, hipertelorismo ocular, puente nasal plano, hendidura labial unilateral completa, hendidura palatina completa, pabellones auriculares displásicos de implantación baja rotada y manos con inclusión del pulgar

Figura 3. Cariotipo

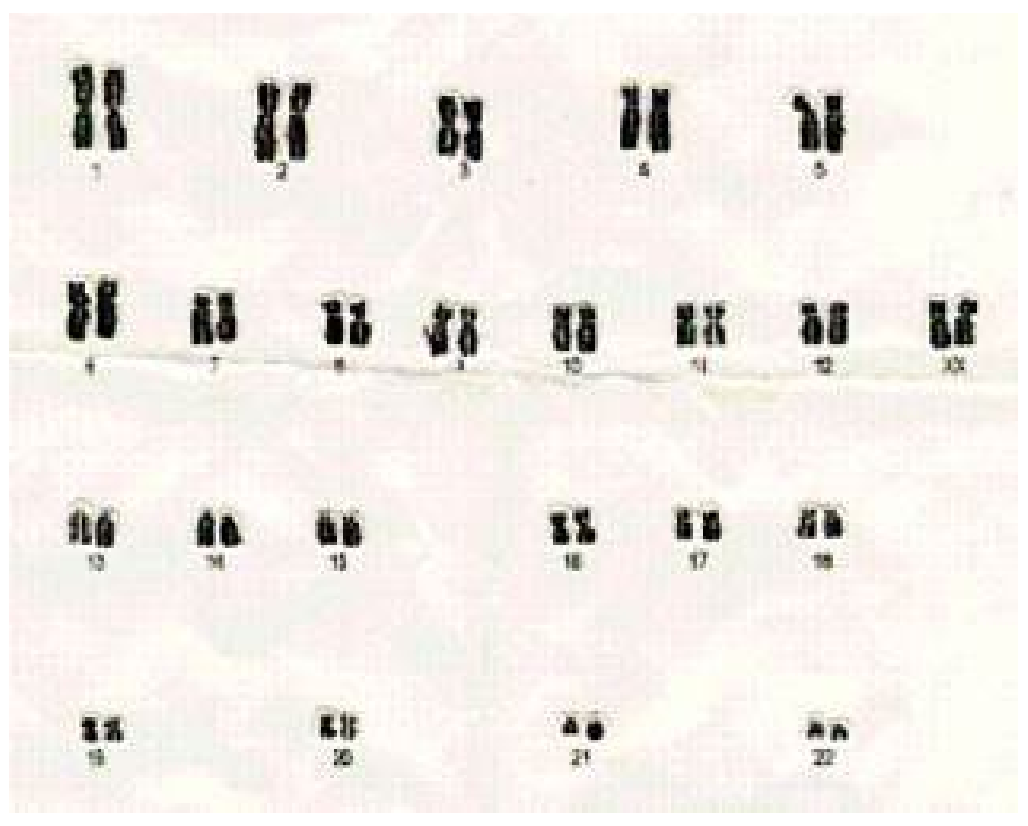

Cariotipo de la paciente presentada, en el cual no se observa ninguna alteración estructural o numérica. Complemento 46, XX.

En 1964 se clasificó la holoprosencefalia en cuatro tipos: alobar, semilobar, lobar y una variante de fusión media interhemisférica, que depende del grado de separación del prosencéfalo $(3,5)$. En el presente caso presentado según las características fenotípicas evidenciadas y los hallazgos del TAC de cráneo, se diagnosticó holoprosencefalia semilobar, en donde la división de los hemisferios se hace de manera parcial y solo se presenta fusión de la parte anterior del encéfalo, mientras que la parte posterior permanece separada (1).
La etiología de la holoprosencefalia es multifactorial y la mayoría de los casos son esporádicos y de causa desconocida, pues solo el $15 \%$ al $20 \%$ de todos los casos es posible reconocer su etiología. Se han relacionado diferentes anomalías genéticas, cromosómicas, teratógenos y asociaciones sindrómicas, por lo cual debe ofrecerse un estudio citogenético en todos los casos, dado que este puede establecer la presencia de alteraciones cromosómicas que tendrán un impacto importante en 
el riesgo de recurrencia (7). Alrededor el $75 \%$ de los casos en quienes se realiza cariotipo se evidencia un patrón cromosómico normal (6).

En el caso presentado el complemento cromosómico se presentó sin alteraciones estructurales o numéricas, 46,XX. Al analizar la historia clínica se evidenció que los controles prenatales habían sido normales, sin exposición teratogénica y los padres no presentaban antecedentes familiares de holoprosencefalia. Llamó la atención la consanguinidad de los padres, quienes son primos en segundo grado, lo cual implica considerar un patrón de herencia autosómico recesivo. En la literatura se ha descrito que estos casos se puede alcanzar un riesgo de recurrencia de hasta el $6 \%$ (7).

Las mutaciones del gen OTX2 (Siglas del Inglés: orthodenticle, drosophila, homolog of, 2) se han relacionado como causa embriológica, disminuyendo la capacidad inductiva del mesodermo, favoreciendo la migración neuronal anómala hacia las porciones ventrales del primer arco branquial y hacia la segunda bolsa faríngea. Esto conduce a una inducción anómala del dorso ventral del tubo neural, afectando la diferenciación entre el diencéfalo y el telencéfalo, la división de los hemisferios cerebrales y la formación de las vesículas ópticas (8). Las mutaciones del gen OTX2, también afectan la vía de señalización de desarrollo embrionario Sonic hedgehog provocando diversos fenotipos del espectro de la holoprosencefalia (9).

Es importante realizar un diagnóstico diferencial con otras anomalías que comprometen la línea media del sistema nervioso central como lo son la esquizencefalia o la porencefalia. Los métodos de elección para el diagnóstico son la tomografía axial computarizada (TAC) o por medio de resonancia magnética nuclear (RMN) (1). En este caso se realizó TAC de cráneo simple que permite realizar diagnósticos diferenciales y realizar una adecuada clasificación así como correlacionar los hallazgos imagenológicos con los hallazgos fenotípicos del paciente.

\section{Conclusiones}

El pronóstico de la holoprosencefalia depende del grado de fusión, así como de otras malformaciones congénitas concomitantes. Se ha descrito que el $3 \%$ de los fetos que presentan holoprosencefalia sobreviven al parto y la mayoría no tiene una expectativa de vida superior a los 6 meses, lo cual resalta la necesidad del diagnóstico temprano prenatal para ofrecer acompañamiento multidisciplinario que incluya el asesoramiento genético.

\section{Agradecimientos}

Agradecemos al Doctor José Dorado por su valioso apoyo y contribución intelectual para la elaboración de este artículo.

\section{Conflictos de interés}

Los autores declaran no existió conflicto de interés en la elaboración del manuscrito.

\section{R E F E R E N C I A S}

1. Sadot Linero T, A. S. L. T., \& Velasco, M. V. (2006). Reporte de un caso clínico de holoprosencefalia. Red De Revistas Científicas De América Latina, El Caribe, España Y Portugal, (Entramado, vol. 2, núm. 2,), pp. 74-81.

2. Castañeyra-Perdomo, A., González-Marrero, I., GonzálezToledo, J., Hernández-Abad, L., Castañeyra-Ruiz, L., GonzalezArnay, E. and Carmona-Calero, E. (2019). A case of holoprosencephaly and a little review. Human Pathology: Case Reports, 15, pp.29-32.

3. Raam, M., Solomon, B. and Muenke, M. (2011). Holoprosencephaly: A guide to diagnosis and clinical management. Indian Pediatrics, 48(6), pp.457-466.

4. Nazer Herrera, J., Cifuentes Ovalle, L. and Cortez López, A. (2015). ECLAMC: 41 años de vigilancia de la holoprosencefalia en Chile. Período 1972-2012. Revista médica de Chile, 143(7), pp.874-879.

5. Hernández, J., Rueda, H. and Hernández, L. (2008). Descripción clínica y anatomopatológica de dos casos de holoprosencefalia. Revista de los estudiantes de la Universidad industrial de Santander.

6. Zatarain-Gulmar A, Ramírez-Vilchis AN (2018). Holoprosencefalia semilobar en una paciente de 1 año y 9 meses de edad: reporte de un caso. Anales de Radiología México.

7. Cañete, P., Lizán, C., Marcos, B., Balanzá, R. and Pellicer, A. (2009). Holoprosencefalia semilobular en 2 gestaciones sucesivas. Clínica e Investigación en Ginecología y Obstetricia, 36(4), pp.148-152.

8. Schiffer C, Tariverdian G, Schiesser M, Thomas MC, Sergi C. Agnathia-otocephaly complex: report of three cases with involvement of two different Carnegie stages. Am J Med Genet 2002; 112: 203-208.

9. Martínez-Frías ML, Bermejo E. Frequency and trends of congenital defects in Spain: usefulness and significance of different frequencies. Med Clin (Barcelona) 1999; 113: 459-462 